\title{
Post-transplant lymphoproliferative disease following liver transplantation
}

\author{
Kala Y. Kamdar ${ }^{1}$, Cliona M. Rooney ${ }^{1,2,3,4}$, and Helen E. Heslop ${ }^{1,4,5}$ \\ ${ }^{1}$ Department of Pediatrics, Baylor College of Medicine, Houston, TX \\ ${ }^{2}$ Department of Molecular Virology and Microbiology, Baylor College of Medicine, Houston, TX \\ ${ }^{3}$ Department of Immunology, Baylor College of Medicine, Houston, TX \\ ${ }^{4}$ Center for Cell and Gene Therapy, Baylor College of Medicine, The Methodist Hospital and \\ Texas Children's Hospital, Houston, TX \\ ${ }^{5}$ Department of Medicine, Baylor College of Medicine, Houston, TX
}

\begin{abstract}
Purpose of review-Despite contemporary immunosuppressive regimens, post-transplant lymphoproliferative disease (PTLD) remains a major complication after liver transplantation. This review highlights advances in the understanding of the pathophysiology, diagnosis, and management of PTLD in liver transplant recipients.

Recent findings-The spectrum of PTLD after liver transplant ranges from polymorphic lymphoproliferation to high grade monoclonal lymphoma and is usually related to outgrowth of lymphocytes infected with Epstein-Barr virus (EBV). Risk factors for PTLD include EBVseronegativity of the recipient, young age, intensity of immunosuppression, and the first year posttransplant. Measurement of EBV load by quantitative polymerase chain reaction assays is an important aid in the surveillance and diagnosis of PTLD although the specificity for PTLD is only about 50\% (specificity for EBV is $\sim 100 \%$ ). In patients diagnosed with PTLD, management options include reduction of immunosuppression, rituximab, combination chemotherapy, and adoptive immunotherapy. Outcomes have improved since rituximab has been incorporated into treatment regimens, and immunotherapy approaches show promise.
\end{abstract}

Summary-PTLD is a significant complication after liver transplantation, particularly in children. Advances in early detection approaches have aided in the diagnosis and management of PTLD, but further research to identify better predictive biomarkers is needed to improve riskbased treatment strategies.

\section{Keywords}

post-transplant lymphoproliferative disease; liver transplantation; Epstein-Barr virus

\section{Introduction}

Post-transplant lymphoproliferative disease (PTLD) occurs in immunosuppressed liver transplant recipients due to uncontrolled lymphoproliferation, which is prevented in

Correspondence to: Kala Y. Kamdar, MD, Texas Children's Cancer Center, 1102 Bates Street, Suite 1220, Houston, TX 77030, Phone 832-824-4163, Fax 832-825-4039, kykamdar@txccc.org.

Financial disclosures:

The authors have no financial disclosures to report. 
immunocompetent individuals by an Epstein-Barr virus (EBV) specific immune response. The PTLD spectrum ranges from reactive, polyclonal hyperplasia to high-grade monoclonal lymphoma and is usually related to outgrowth of EBV-infected lymphocytes [1]. The incidence of PTLD is increased in individuals receiving intensive immunosuppression. The incidence is also higher in organs with many B-cells, such as small bowel [2]. In liver transplant recipients, PTLD is reported in up to $2.8 \%$ of adults and up to $15 \%$ of children [3]. Mortality has been reported as high as 50\%, although current management strategies appear to result in improved outcomes [4-7]. Here we will review the pathophysiology, risk factors, clinical presentation, and management of PTLD after liver transplantation.

\section{Pathophysiology of PTLD}

Although PTLD is heterogeneous, EBV has a critical role in its pathogenesis.

Approximately $85 \%$ of PTLD is of B-cell origin, and more than $80 \%$ of these are associated with EBV [8]. Of the $15 \%$ that is of T-cell origin, $30 \%$ are associated with EBV [9].

\section{EBV-positive PTLD}

EBV is a $\gamma$-herpesvirus with the ability to stimulate B-cell proliferation and transformation. In normal individuals, primary lytic EBV infection occurs in the oropharynx and triggers a robust humoral and cellular immune response. After clearance of the primary infection, EBV persists in infected B-lymphocytes, establishing latent infection. Four types of latency exist, differentiated by the EBV antigen expression pattern in infected B-cells. In type 3 latency, six nuclear proteins (EBNA 1-6), two membrane proteins (LMP 1-2), and two EBV-encoded RNAs (EBER 1-2) are expressed, driving B-cell proliferation and memory Bcell expansion. Type 3 latency is highly immunogenic and rarely detected in immunocompetent individuals. In type 2 latency, EBNA 1 and LMP 1-2 are expressed and drive B-cell differentiation into the memory compartments in germinal centers. Upon entering the peripheral circulation, infected memory B-cells downregulate viral proteins. Type 1 latency is associated with expression of only EBNA 1 and is seen in proliferating Bcells. Most infected circulating B-cells express no viral antigens, type 0 latency, and remain invisible to the host immune system. Reactivation to type 3 latency likely occurs periodically but is rapidly controlled by the EBV-specific cytotoxic T-lymphocyte (CTL) response [10-13]. Pharmacologic immunosuppression after transplantation leads to decreased frequency and functional impairment of EBV-specific CTLs [14]. In this setting, EBV-driven lymphoproliferation with type 3 latency may occur and is labeled PTLD, with PTLD depending on the virus to inhibit apoptosis and drive proliferation [12;15*]. However, the progression to PTLD may also require molecular alterations, such as microsatellite instability, DNA hypermethylation, aberrant somatic hypermutation, or alterations of c-myc, bcl-6, and p53 [16-18].

\section{EBV-negative PTLD}

The mechanism behind the development of EBV-negative PTLD is unclear, but it is usually associated with later onset, monomorphic histology, and aggressive clinical behavior [17;19-21**]. EBV-negative PTLD may be associated with as yet unidentified viral agents or loss of EBV. In the latter hit-and-run scenario, lymphoproliferation initially stimulated by EBV may lead to new mutations over time, including alterations that result in EBVindependent cell replication [22].

\section{Risk Factors for PTLD}

Risk factors for PTLD after liver transplant include EBV-seronegativity in the recipient, age $\geq 18$ years, degree of immunosuppression, and first year post-transplant [3;4;23-26]. EBV-seronegative patients who receive a transplant from an EBV-seropositive donor are at 
particularly high risk, because EBV-infected B-cells in the transplanted organ are invariably introduced into the recipient. The recipient has neither virus-specific antibodies to neutralize infectious virions released by infected B-cells nor virus-specific T-cells to control the outgrowth of subsequently infected recipient B-cells. An increased incidence of PTLD is also seen with intensive immunosuppressive regimens using anti T-cell antibodies such as OKT3 and ATG [4]. It has been suggested that mTOR inhibitors may be associated with decreased incidence of PTLD [27], but clinical studies have been inconclusive, partly due to lack of widespread use [28-30]. Recipients who receive steroids pre-transplant due to immunological disorders may have an increased risk of PTLD [31].

\section{Clinical Presentation of PTLD}

The 2008 World Health Organization classification of PTLD includes four categories (Table 1). Early lesions usually occur within one year of transplantation and may have features of reactive plasmacytic hyperplasia or infectious mononucleosis [1]. These EBV-driven lesions are frequently polyclonal, and they are more common in patients who were EBVseronegative pre-transplant and in younger patients. Polymorphic PTLD, monomorphic PTLD, and classic Hodgkin lymphoma-type PTLD are more likely to be monoclonal and have variable onset after transplantation. Monomorphic B-cell PTLD, particularly diffuse large B-cell lymphoma, is the most common. [1;32;33*]. Monomorphic and monoclonal PTLD may be more aggressive, but histology and clonality do not consistently predict outcome [1].

Post-transplant lymphomas are more likely than general lymphomas to have extranodal involvement, high grade, aggressive clinical behavior, and poor outcomes. Poor prognostic factors for PTLD include high grade, poor performance status, EBV-negativity, and graft involvement [5**]. Symptoms may include fever, lymphadenopathy, weight loss, or splenomegaly. Disease may be localized to one site or to the allograft, or it may present as diffuse disease with multi-organ failure. Bone marrow and central nervous system (CNS) involvement may also occur $\left[21^{* * ;} ; 24 ; 32 ; 34\right]$.

Post-transplant lymphomas in liver transplant recipients have preference for localization to the liver. The Collaborative Transplant Study includes 165 liver transplant recipients with PTLD. Of these, $21.8 \%$ had disease localized to the liver, whereas $13.3 \%$ demonstrated multifocal disease [4].

\section{Diagnosis of PTLD}

Early diagnosis of PTLD is important to facilitate prompt initiation of treatment and prevent evolution to a more aggressive variant. Guidelines for the diagnosis and management of PTLD in adult solid organ transplant recipients were developed by the British Committee for Standards in Haematology and the British Transplantation Society and published in the last year [ $\left.5^{* *} ; 21^{* *}\right]$. Similar guidelines for children have not yet been developed, but the benefits of early diagnosis are illustrated by a recent comparison of PTLD in liver transplant recipients at a single institution during 1993-1997 and 2001-2005. In the latter period, patients had less advanced histological disease at presentation and no deaths, compared to advanced disease and 3 deaths in the earlier era. The difference was ascribed to implementation of molecular EBV monitoring and preemptive reduction in immunosuppression [7].

A high index of suspicion is essential to early detection of PTLD. Measurement of EBV load by quantitative polymerase chain reaction assays is an important aid in surveillance and diagnosis of PTLD. A number of assays that use whole blood, serum, or peripheral blood mononuclear cells (PBMCs) are available but require different interpretation [35]. A virus 
load in PBMCs reflects the frequency of virus-infected cells, while a virus load in serum can reflect free virus DNA released by killed infected cells or infectious virions released by productively infected cells. The latter two possibilities can be distinguished by treatment of serum with DNAse, which destroys free viral DNA while DNA in virions is protected by the capsid [36]. A high virus load in whole blood may result from any of the three possibilities. Although the frequency of testing EBV load post-transplant has not been established, close monitoring in pediatric transplant recipients during the first year post-transplant is recommended [13**;37]. While EBV load is highly sensitive for predicting PTLD, the specificity in liver transplant recipients is only around $50 \%\left[5^{* *} ; 21^{* *}\right]$, which may reflect increased numbers of EBV-infected cells with restricted latency in some recipients [38]. Nevertheless, longitudinal monitoring of EBV DNA can help differentiate patients with a persistently elevated but stable EBV load from those with an increasing EBV load. Because of the low specificity of EBV load, there is interest in identifying additional biomarkers for PTLD risk. Alternatives, such as soluble CD30, are being evaluated [39]. Combining assays of T-cell function with viral load may also improve predictive power [40].

A progressively rising EBV DNA load should prompt further investigation for PTLD by physical examination and imaging [13**;41]. If clinical suspicion is raised, a low threshold should be used for pursuing tissue biopsy. Excisional biopsy is strongly preferred over needle core biopsy, and fine needle aspiration is inadequate for diagnosis. Tissue should be submitted for EBV analysis (usually EBER in situ hybridization or immunohistochemistry for the LMP1 antigen) and determination of CD20 expression, which are useful in determining treatment options. If PTLD is diagnosed, a comprehensive staging and pretreatment workup is necessary, including: CT of neck, chest, abdomen/pelvis; evaluation of the transplanted liver; echocardiogram and glomerular filtration rate. Fluorodeoxyglucosepositron emission tomography, particularly when combined with CT, can identify areas infiltrated by lymphoma that have not yet increased in size [24]. Some patients may need bone marrow evaluation, CNS imaging, or lumbar puncture.

\section{Preemptive Therapy for PTLD}

The clinical context and the risk of PTLD are important in deciding when to institute preemptive therapy. The intensity of immunosuppression is a modifiable risk factor for PTLD, so reduction of immunosuppression may be considered if a rising EBV load is seen early after transplantation in the setting of high immunosuppression. In 73 pediatric liver transplant patients treated during 2001-2004, the incidence of PTLD was reduced from 16\% to $2 \%$ by reducing immunosuppression in individuals with a high EBV load. Only one patient developed graft rejection [42]. Clearly, the risk of graft rejection must be considered before reduction in immunosuppression. The liver is a tolerogenic organ, with a low incidence of graft losses from acute or chronic rejection. Reduction of immunosuppression is tolerated better after liver transplant than other solid organ transplants, with withdrawal of immunosuppression possible in selected patients [43].

Antiviral therapy may help prevent PTLD in EBV-seronegative solid organ transplant recipients. These agents can block EBV replication in donor B-cells and infection of recipient B-cells [44-46]. Indeed, prophylactic intravenous ganciclovir after liver transplant in children has been associated with decreased incidence of PTLD [44], perhaps due to reduction in the number of latently-infected B-lymphocytes [10;45]. However, antiviral agents have no effect on the growth of established PTLD when the cells are already transformed. 


\section{Treatment of PTLD}

Treatment options for established PTLD include reduction of immunosuppression, targeting of B-cells with monoclonal antibodies, or chemotherapy. Surgery may be an option for localized disease. Treatment decisions are based on histology, stage, location, clinical status, graft status, and time after transplant.

\section{Reduction in Immunosuppression}

Reduction of immunosuppression can lead to regression of PTLD due to restoration of Tcell functions. Immunosuppression should be decreased to the lowest tolerated levels, which are often $25-50 \%$ of the starting dose, depending on concerns for graft rejection. In some patients, reducing immunosuppression alone is sufficient to produce remission, while in others it is used as adjunct treatment. Risk factors for failure of immunosuppression reduction include high lactate dehydrogenase (LDH), organ dysfunction, and multiorgan involvement at diagnosis. In individuals without any of these risk factors, a $90 \%$ response rate has been seen with reduction of immunosuppression [47].

The response to reduction in immunosuppression is usually seen within 2-4 weeks and should be assessed by evaluating the change in tumor size, LDH, and EBV load [47]. When immunosuppression cannot be reduced or reduction is inadequate, alternative therapies should be considered early. Early lesions and polymorphic lymphomas often respond well to immunosuppression reduction. Late-onset, monomorphic PTLD is less responsive to immunosuppression reduction and may need rituximab and/or chemotherapy.

\section{Rituximab}

Rituximab is a monoclonal antibody against CD20, a B-lymphocyte specific antigen frequently expressed on PTLD. Rituximab monotherapy appears to be effective in the management of selected B-cell, CD20-positive PTLD cases, with remission rates of 44\%$65 \%$ [48-51]. A multicenter, retrospective analysis suggests that both progression-free and overall survival are significantly improved with early rituximab-based treatment in PTLD [6*]. However, some patients may progress on or after rituximab, necessitating chemotherapy [52].

\section{Chemotherapy}

Anthracycline-based chemotherapy for PTLD is effective in achieving long-term diseasefree survival, although the benefits must be weighed against potential toxicities. The use of CHOP (cyclophosphamide, doxorubicin, vincristine, and prednisone), with or without rituximab, has response rates of $65-100 \%$ with very low rates of graft loss [50;53-55]. Prompt institution of CHOP-based therapy should be used for patients with high-grade lymphoma, inadequate response or progression despite other therapy, or critical organ compromise. Late-onset PTLD outcomes may be better with CHOP-based regimens. A less intensive chemotherapy regimen with cyclophosphamide and prednisone also has excellent response rates, up to $83 \%$ in children, and might be considered as an alternative [56;57].

\section{Local Control}

In the minority of liver PTLDs that are localized at presentation, surgical resection or radiotherapy may be useful in conjunction with immunosuppression reduction. However, the

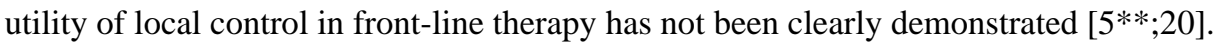
Surgery and radiation may be helpful in the management of local complications from PTLD, and radiation may be considered for certain disease locations such as orbit or CNS [5*;56]. Primary CNS lymphoma after transplant is very difficult to treat, with mortality up to $88 \%$ 
[58]. High-dose methotrexate chemotherapy has also shown activity in children with CNS PTLD after liver transplant [59].

\title{
Adoptive Immunotherapy
}

Adoptive immunotherapy with EBV-specific CTLs to restore a cellular immune response to EBV is theoretically an attractive treatment option. Infusions of autologous EBV-specific CTLs generated from peripheral blood of the recipient have been associated with a marked decrease in EBV DNA [60] and have been given without significant toxicity [61]. However, long-term EBV-specific immunity is not sustained in patients, such as solid organ transplant recipients, who continue to receive immunosuppression. The generation of autologous EBVspecific CTLs is also time- and labor-intensive, requires specialized facilities, and is currently available only through investigational trials.

Infusion of partially human leukocyte antigen-matched CTLs derived from a bank of EBVseropositive donors may allow timely administration of immunotherapy to more patients [62;63]. This approach resulted in a 52\% response rate at 6 months in patients who had received solid organ transplant or hematopoietic stem cell transplant [64]. A recent update 4-9 years after CTL infusion showed increased survival among recipients who attained a partial or complete response. Of the 14 patients who achieved an initial complete response, only 1 subsequently died of relapse [65*].

\section{Conclusion}

A general strategy for monitoring and treating PTLD is shown in Figure 1. We recommend monitoring of EBV DNA in high-risk liver transplant recipients at 1-2 week intervals. Patients with rising EBV DNA but no signs of PTLD may be considered for preemptive therapy, particularly reduction in immunosuppression. Indeed, a high virus load may be used as a marker of overimmunosuppression [42]. In patients with clinical findings suggestive of PTLD, biopsy should be obtained and staging/pre-treatment studies should be performed. Immunosuppression should be reduced if possible, and further management should be based on histology, stage, location, and performance status. Surgical resection and/or radiotherapy may be considered for localized stage I disease. Patients with clinically low risk B-cell disease may be treated with rituximab monotherapy. Patients with failure to respond to the above measures, high risk disease, or critical organ compromise should urgently be considered for chemotherapy. Clinical trials using EBV-specific CTLs may be considered for these patients if available. Prospective studies are needed to develop prediction models for PTLD risk and improve risk-stratified treatment algorithms for PTLD.

\section{Acknowledgments}

\author{
Funding support:
}

This work was supported by NIH grants PO1 CA94237, P50CA126752, and U54HL081007; a Specialized Center of Research Award from the Leukemia \& Lymphoma Society; and Production Assistance for Cellular Therapies (NHLBI) contract number HHSN268201000007C.

\section{References}

1. Swerdlow, SH.; Webber, SA.; Chadburn, A.; Ferry, J. Classification of Tumours of Haematopoietic and Lymphoid Tissues. 4. Swerdlow, SH.; Campo, E.; Harris, NL., editors. Lyon, France: International Agency for Research on Cancer; 2008.

2. Allen UD, Farkas G, Hebert D, et al. Risk factors for post-transplant lymphoproliferative disorder in pediatric patients: a case-control study. Pediatr Transplant. 2005; 9:450-455. [PubMed: 16048596] 
3. Taylor AL, Marcus R, Bradley JA. Post-transplant lymphoproliferative disorders (PTLD) after solid organ transplantation. Crit Rev Oncol Hematol. 2005; 56:155-167. [PubMed: 15979320]

4. Opelz G, Dohler B. Lymphomas after solid organ transplantation: a collaborative transplant study report. Am J Transplant. 2004; 4:222-230. [PubMed: 14974943]

**5. Parker A, Bowles K, Bradley JA, et al. Management of post-transplant lymphoproliferative disorder in adult solid organ transplant recipients - BCSH and BTS Guidelines. Br J Haematol. 2010; 149:693-705. These guidelines for managing PTLD were developed by the British Committee for Standards in Haematology and the British Transplantation Society. [PubMed: 20408848]

*6. Evens AM, David KA, Helenowski I, et al. Multicenter analysis of 80 solid organ transplantation recipients with post-transplantation lymphoproliferative disease: outcomes and prognostic factors in the modern era. J Clin Oncol. 2010; 28:1038-1046. This multicenter study showed improved outcomes in PTLD since the initiation of early rituximab-based treatment. [PubMed: 20085936]

7. Kerkar N, Morotti RA, Madan RP, et al. The changing face of post-transplant lymphoproliferative disease in the era of molecular EBV monitoring. Pediatr Transplant. 2010; 14:504-511. [PubMed: 20070559]

8. Allen U, Alfieri C, Preiksaitis J, et al. Epstein-Barr virus infection in transplant recipients: Summary of a workshop on surveillance, prevention and treatment. Can J Infect Dis. 2002; 13:89-99. [PubMed: 18159378]

9. Hoshida Y, Li T, Dong Z, et al. Lymphoproliferative disorders in renal transplant patients in Japan. Int J Cancer. 2001; 91:869-875. [PubMed: 11275994]

10. Thorley-Lawson DA, Gross A. Persistence of the Epstein-Barr virus and the origins of associated lymphomas. N Engl J Med. 2004; 350:1328-1337. [PubMed: 15044644]

11. Straathof KC, Savoldo B, Heslop HE, Rooney CM. Immunotherapy for post-transplant lymphoproliferative disease. Br J Haematol. 2002; 118:728-740. [PubMed: 12181039]

12. Gottschalk S, Rooney CM, Heslop HE. Post-transplant lymphoproliferative disorders. Annu Rev Med. 2005; 56:29-44. [PubMed: 15660500]

**13. Heslop HE. How I treat EBV lymphoproliferation. Blood. 2009; 114:4002-4008. This paper provides an overview of the biology and treatment strategies for PTLD. [PubMed: 19724053]

14. Smets F, Sokal EM. Epstein-Barr virus-related lymphoproliferation in children after liver transplant: role of immunity, diagnosis, and management. Pediatr Transplant. 2002; 6:280-287. [PubMed: 12234267]

*15. Vereide DT, Sugden B. Lymphomas differ in their dependence on Epstein-Barr virus. Blood. 2010 In this study, the authors conditionally evicted the viral extrachromosomal genome from tumor cells in vitro to examine the role of EBV in different lymphomas and showed that PTLD cells depend on the virus to inhibit apoptosis.

16. Capello D, Rossi D, Gaidano G. Post-transplant lymphoproliferative disorders: molecular basis of disease histogenesis and pathogenesis. Hematol Oncol. 2005; 23:61-67. [PubMed: 16216037]

17. Nelson BP, Nalesnik MA, Bahler DW, et al. Epstein-Barr virus-negative post-transplant lymphoproliferative disorders: a distinct entity? Am J Surg Pathol. 2000; 24:375-385. [PubMed: 10716151]

18. Vakiani E, Basso K, Klein U, et al. Genetic and phenotypic analysis of B-cell post-transplant lymphoproliferative disorders provides insights into disease biology. Hematol Oncol. 2008; 26:199-211. [PubMed: 18457340]

19. Dotti G, Fiocchi R, Motta T, et al. Epstein-Barr virus-negative lymphoproliferate disorders in longterm survivors after heart, kidney, and liver transplant. Transplantation. 2000; 69:827-833. [PubMed: 10755535]

20. Dotti G, Fiocchi R, Motta T, et al. Lymphomas occurring late after solid-organ transplantation: influence of treatment on the clinical outcome. Transplantation. 2002; 74:1095-1102. [PubMed: 12438953]

**21. Parker A, Bowles K, Bradley JA, et al. Diagnosis of post-transplant lymphoproliferative disorder in solid organ transplant recipients - BCSH and BTS Guidelines. Br J Haematol. 2010; 149:675692. These guidelines for diagnosing PTLD were developed by the British Committee for Standards in Haematology and the British Transplantation Society. [PubMed: 20408847] 
22. Gan YJ, Razzouk BI, Su T, Sixbey JW. A defective, rearranged Epstein-Barr virus genome in EBER-negative and EBER-positive Hodgkin's disease. Am J Pathol. 2002; 160:781-786. [PubMed: 11891176]

23. Dharnidharka VR, Tejani AH, Ho PL, Harmon WE. Post-transplant lymphoproliferative disorder in the United States: young Caucasian males are at highest risk. Am J Transplant. 2002; 2:993998. [PubMed: 12482154]

24. Bakker NA, van Imhoff GW, Verschuuren EA, van Son WJ. Presentation and early detection of post-transplant lymphoproliferative disorder after solid organ transplantation. Transpl Int. 2007; 20:207-218. [PubMed: 17291214]

25. Birkeland SA, Hamilton-Dutoit S. Is posttransplant lymphoproliferative disorder (PTLD) caused by any specific immunosuppressive drug or by the transplantation per se? Transplantation. 2003; 76:984-988. [PubMed: 14508366]

26. Ison MG, Hager J, Blumberg E, et al. Donor-derived disease transmission events in the United States: data reviewed by the OPTN/UNOS Disease Transmission Advisory Committee. Am J Transplant. 2009; 9:1929-1935. [PubMed: 19538493]

27. El-Salem M, Raghunath PN, Marzec M, et al. Constitutive activation of mTOR signaling pathway in post-transplant lymphoproliferative disorders. Lab Invest. 2007; 87:29-39. [PubMed: 17075574]

28. Jaber JJ, Feustel PJ, Elbahloul O, et al. Early steroid withdrawal therapy in renal transplant recipients: a steroid-free sirolimus and CellCept-based calcineurin inhibitor-minimization protocol. Clin Transplant. 2007; 21:101-109. [PubMed: 17302598]

29. Caillard S, Dharnidharka V, Agodoa L, et al. Posttransplant lymphoproliferative disorders after renal transplantation in the United States in era of modern immunosuppression. Transplantation. 2005; 80:1233-1243. [PubMed: 16314791]

30. Khwaja K, Asolati M, Harmon J, et al. Outcome at 3 years with a prednisone-free maintenance regimen: a single-center experience with 349 kidney transplant recipients. Am J Transplant. 2004; 4:980-987. [PubMed: 15147433]

31. Zimmermann T, Hoppe-Lotichius M, Tripkovic V, et al. Liver transplanted patients with preoperative autoimmune hepatitis and immunological disorders are at increased risk for PostTransplant Lymphoproliferative Disease (PTLD). Eur J Intern Med. 2010; 21:208-215. [PubMed: 20493424]

32. Koch DG, Christiansen L, Lazarchick J, et al. Posttransplantation lymphoproliferative disorder--the great mimic in liver transplantation: appraisal of the clinicopathologic spectrum and the role of Epstein-Barr virus. Liver Transpl. 2007; 13:904-912. [PubMed: 17539010]

*33. Rinaldi A, Capello D, Scandurra M, et al. Single nucleotide polymorphism-arrays provide new insights in the pathogenesis of post-transplant diffuse large B-cell lymphoma. Br J Haematol. 2010; 149:569-577. In this study, the authors perform SNP arrays and show that PTLD with DLBCL morphology has small interstitial deletions targeting known fragile sites, such as FRA1B, FRA2E, and FRA3B. [PubMed: 20230398]

34. Mucha K, Foroncewicz B, Ziarkiewicz-Wroblewska B, et al. Post-transplant lymphoproliferative disorder in view of the new WHO classification: a more rational approach to a protean disease? Nephrol Dial Transplant. 2010; 25:2089-2098. [PubMed: 20576725]

*35. Gulley ML, Tang W. Using Epstein-Barr viral load assays to diagnose, monitor, and prevent posttransplant lymphoproliferative disorder. Clin Microbiol Rev. 2010; 23:350-366. This paper provides an overview of assays of EBV viral load to detect PTLD. [PubMed: 20375356]

36. Ambinder RF. Posttransplant lymphoproliferative disease: pathogenesis, monitoring, and therapy. Curr Oncol Rep. 2003; 5:359-363. [PubMed: 12895385]

37. Stevens SJ, Verschuuren EA, Pronk I, et al. Frequent monitoring of Epstein-Barr virus DNA load in unfractionated whole blood is essential for early detection of posttransplant lymphoproliferative disease in high-risk patients. Blood. 2001; 97:1165-1171. [PubMed: 11222357]

38. Gotoh K, Ito Y, Ohta R, et al. Immunologic and virologic analyses in pediatric liver transplant recipients with chronic high Epstein-Barr virus loads. J Infect Dis. 2010; 202:461-469. [PubMed: 20560768] 
39. Haque T, Chaggar T, Schafers J, et al. Soluble CD30: a serum marker for Epstein-Barr virusassociated lymphoproliferative diseases. J Med Virol. 2011; 83:311-316. [PubMed: 21181928]

40. Lee TC, Goss JA, Rooney CM, et al. Quantification of a low cellular immune response to aid in identification of pediatric liver transplant recipients at high-risk for EBV infection. Clin Transplant. 2006; 20:689-694. [PubMed: 17100717]

41. Bakker NA, van Imhoff GW. Post-transplant lymphoproliferative disorders: from treatment to early detection and prevention? Haematologica. 2007; 92:1447-1450. [PubMed: 18024391]

42. Lee TC, Savoldo B, Rooney CM, et al. Quantitative EBV viral loads and immunosuppression alterations can decrease PTLD incidence in pediatric liver transplant recipients. Am J Transplant. 2005; 5:2222-2228. [PubMed: 16095501]

43. Londono MC, Lopez MC, Sanchez-Fueyo A. Minimization of immunosuppression in adult liver transplantation: new strategies and tools. Curr Opin Organ Transplant. 2010

44. McDiarmid SV, Jordan S, Kim GS, et al. Prevention and preemptive therapy of postransplant lymphoproliferative disease in pediatric liver recipients. Transplantation. 1998; 66:1604-1611. [PubMed: 9884246]

45. Hierro L, ez-Dorado R, Diaz C, et al. Efficacy and safety of valganciclovir in liver-transplanted children infected with Epstein-Barr virus. Liver Transpl. 2008; 14:1185-1193. [PubMed: 18668670]

46. Funch DP, Walker AM, Schneider G, et al. Ganciclovir and acyclovir reduce the risk of posttransplant lymphoproliferative disorder in renal transplant recipients. Am J Transplant. 2005; 5:2894-2900. [PubMed: 16303002]

47. Tsai DE, Nearey M, Hardy CL, et al. Use of EBV PCR for the diagnosis and monitoring of posttransplant lymphoproliferative disorder in adult solid organ transplant patients. Am J Transplant. 2002; 2:946-954. [PubMed: 12482147]

48. Oertel SH, Anagnostopoulos I, Bechstein WO, et al. Treatment of posttransplant lymphoproliferative disorder with the anti-CD20 monoclonal antibody rituximab alone in an adult after liver transplantation: a new drug in therapy of patients with posttransplant lymphoproliferative disorder after solid organ transplantation? Transplantation. 2000; 69:430-432. [PubMed: 10706055]

49. Blaes AH, Peterson BA, Bartlett N, et al. Rituximab therapy is effective for posttransplant lymphoproliferative disorders after solid organ transplantation: results of a phase II trial. Cancer. 2005; 104:1661-1667. [PubMed: 16149091]

50. Jain AB, Marcos A, Pokharna R, et al. Rituximab (chimeric anti-CD20 antibody) for posttransplant lymphoproliferative disorder after solid organ transplantation in adults: long-term experience from a single center. Transplantation. 2005; 80:1692-1698. [PubMed: 16378063]

51. Choquet S, Leblond V, Herbrecht R, et al. Efficacy and safety of rituximab in B-cell posttransplantation lymphoproliferative disorders: results of a prospective multicenter phase 2 study. Blood. 2006; 107:3053-3057. [PubMed: 16254143]

52. Savoldo B, Rooney CM, Quiros-Tejeira RE, et al. Cellular immunity to Epstein-Barr virus in liver transplant recipients treated with rituximab for post-transplant lymphoproliferative disease. Am J Transplant. 2005; 5:566-572. [PubMed: 15707412]

53. Taylor AL, Bowles KM, Callaghan CJ, et al. Anthracycline-based chemotherapy as first-line treatment in adults with malignant posttransplant lymphoproliferative disorder after solid organ transplantation. Transplantation. 2006; 82:375-381. [PubMed: 16906036]

54. Choquet S, Oertel S, Leblond V, et al. Rituximab in the management of post-transplantation lymphoproliferative disorder after solid organ transplantation: proceed with caution. Ann Hematol. 2007; 86:599-607. [PubMed: 17522862]

55. Buadi FK, Heyman MR, Gocke CD, et al. Treatment and outcomes of post-transplant lymphoproliferative disease: a single institution study. Am J Hematol. 2007; 82:208-214. [PubMed: 17022049]

56. Buell JF, Gross TG, Hanaway MJ, et al. Posttransplant lymphoproliferative disorder: significance of central nervous system involvement. Transplant Proc. 2005; 37:954-955. [PubMed: 15848587]

57. Orjuela M, Gross TG, Cheung YK, et al. A pilot study of chemoimmunotherapy (cyclophosphamide, prednisone, and rituximab) in patients with post-transplant 
lymphoproliferative disorder following solid organ transplantation. Clin Cancer Res. 2003; 9:3945S-3952S. [PubMed: 14506193]

58. Penn I, Porat G. Central nervous system lymphomas in organ allograft recipients. Transplantation. 1995; 59:240-244. [PubMed: 7839447]

59. Taj MM, Messahel B, Mycroft J, et al. Efficacy and tolerability of high-dose methotrexate in central nervous system positive or relapsed lymphoproliferative disease following liver transplant in children. Br J Haematol. 2008; 140:191-196. [PubMed: 18173755]

60. Comoli P, Labirio M, Basso S, et al. Infusion of autologous Epstein-Barr virus (EBV)-specific cytotoxic T cells for prevention of EBV-related lymphoproliferative disorder in solid organ transplant recipients with evidence of active virus replication. Blood. 2002; 99:2592-2598. [PubMed: 11895798]

61. Savoldo B, Goss JA, Hammer MM, et al. Treatment of solid organ transplant recipients with autologous Epstein Barr virus-specific cytotoxic T lymphocytes (CTLs). Blood. 2006; 108:29422949. [PubMed: 16835376]

62. Haque T, Wilkie GM, Taylor C, et al. Treatment of Epstein-Barr-virus-positive posttransplantation lymphoproliferative disease with partly HLA-matched allogeneic cytotoxic T cells. Lancet. 2002; 360:436-442. [PubMed: 12241714]

63. Gandhi MK, Wilkie GM, Dua U, et al. Immunity, homing and efficacy of allogeneic adoptive immunotherapy for posttransplant lymphoproliferative disorders. Am J Transplant. 2007; 7:12931299. [PubMed: 17425621]

64. Haque T, Wilkie GM, Jones MM, et al. Allogeneic cytotoxic T-cell therapy for EBV-positive posttransplantation lymphoproliferative disease: results of a phase 2 multicenter clinical trial. Blood. 2007; 110:1123-1131. [PubMed: 17468341]

*65. Haque T, McAulay KA, Kelly D, Crawford DH. Allogeneic T-cell therapy for Epstein-Barr viruspositive posttransplant lymphoproliferative disease: long-term follow-up. Transplantation. 2010; 90:93-94. This update on long-term follow-up of patients who received third party allogeneic CTLs to treat PTLD showed that only 1 of 14 patients who received a complete response subsequently relapsed. [PubMed: 20606564] 
Key points

- Risk factors for PTLD include EBV-seronegativity of the recipient, young age at transplantation, intensity of immunosuppression, and first year after transplant.

- Measurement of EBV load by quantitative polymerase chain reaction assays is an important aid in the surveillance and diagnosis of PTLD.

- Preemptive therapy, including reduction in immunosuppression and/or antiviral therapy, should be considered in patients at high risk for PTLD.

- PTLD management should be based on the clinical context and the risk profile of the patient and may include reduction in immunosuppression, rituximab, chemotherapy, and/or adoptive immunotherapy 


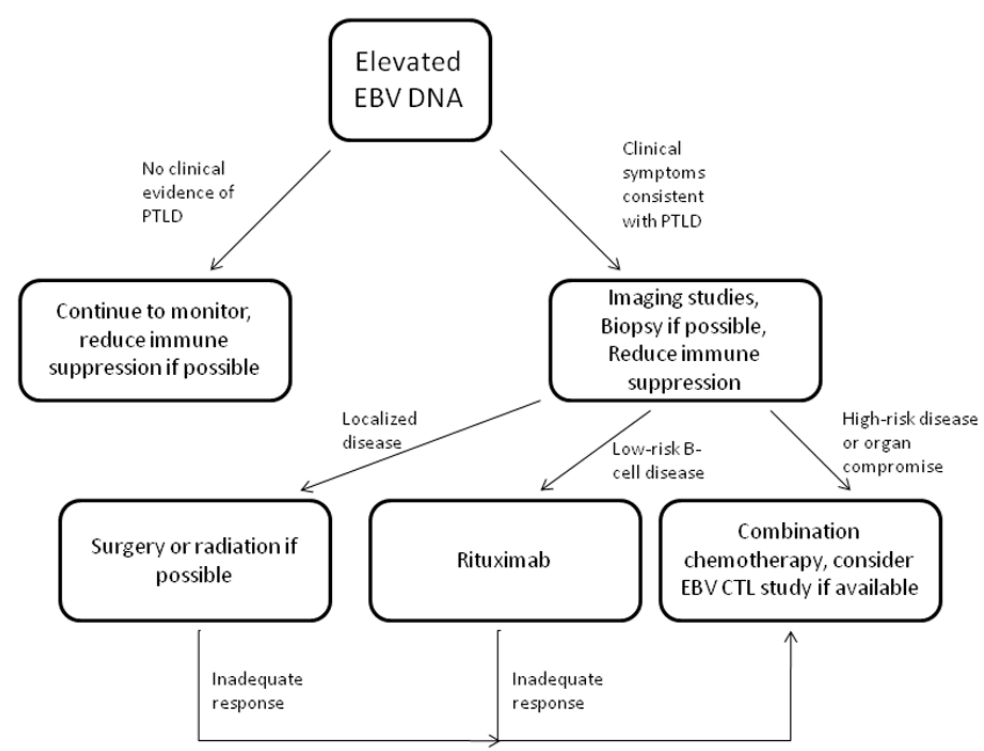

Figure 1.

Monitoring and treatment strategy for PTLD 
Table I

WHO classification of PTLD 2008 [1]

\begin{tabular}{lcc}
\hline Category & Clonality & EBV status \\
\hline Early lesions (plasmacytic hyperplasia, infectious mononucleosis) & Polyclonal & Always EBV+ \\
Polymorphic PTLD & Monoclonal & Always EBV+ \\
Monomorphic PTLD & & \\
B-cell lymphomas & Monoclonal & Frequently EBV+ \\
T-cell lymphomas & Monoclonal & Rarely EBV+ \\
Classic Hodgkin lymphoma-like PTLD & Monoclonal & Frequently EBV+ \\
\hline
\end{tabular}

